\title{
Les subjectivités cosmopolitiques et la question esthétique
}

\author{
Nathalie Blanc, Jacques Lolive ${ }^{1}$
}

Les artistes contemporains qui contribuent à l'espace public, mettent-ils en œuvre une esthétique qui puisse fournir des pistes pour la composition d'un cosmos, d'un monde commun plus qu'humain, l'élaboration d'une cosmopolitique? La perspective esthétique peut-elle faire contrepoids à l'hégémonie scientifique et technique dans le registre politique ? Comment les cosmopolitiques peuvent-elles intégrer une perspective esthétique ? Ces interrogations nous incitent à formuler des éléments théoriques pour la place de l'art dans l'espace public et une esthétique écologique. Nous utilisons la question esthétique comme une modalité riche de passage entre microcosmes et espace public, et les cosmopolitiques comme une manière de penser l'articulation des mondes et une politique. Quelles sont les règles, dès lors, qui permettent de penser cet espace public esthétique?

Notre recherche, aux aspects théoriques aussi bien qu'empiriques, propose d'alimenter la réflexion sur les cosmopolitiques en partant des pratiques artistiques contemporaines dans l'espace public. Notre hypothèse est la suivante : nous pensons que, par fragments, les artistes composent des mondes et réalisent des jonctions entre mondes privés et monde commun. Nous pensons que les modalités du travail artistique fournissent des pistes, sur le plan du jugement esthétique, pour l'élaboration d'une cosmopolitique. C'est à l'intérieur de ce champ d'interrogation et conscients des difficultés mais aussi de l'importance de l'entreprise, que nous avons entrepris de réaliser, dans le cadre d'une réflexion sur la place des artistes et du jugement esthétique dans le registre du politique, des entretiens avec des artistes contemporains intervenant selon des modalités diverses dans l'espace public. Ce travail, dont nous rendons compte ici partiellement, se poursuit aujourd'hui avec des rencontres pour évaluer plus généralement la place de l'esthétique et de la créativité dans le champ de l'écologie.

Nous présenterons d'abord notre problématique générale qui associe cosmopolitique et esthétique (chapitre I), puis nous exposerons notre parcours de recherche. Nous explorerons dans un premier temps la diversité des liens (rationnels mais aussi sensibles et esthétiques) qu'entretiennent les sujets avec leurs mondes singuliers (chapitre II). Selon nous, cette partmonde des sujets si souvent amnésiée constitue pourtant la chair de notre vivre ensemble, la matière vivante de notre monde commun. Malheureusement la prise en compte du sujet et de sa part mondaine par les mécanismes politiques qui composent ce monde commun se heurte à deux obstacles : la clôture territoriale des mondes singuliers et leur éviction par le recours à l'intérêt général et l'expertise scientifique (chapitre III). La perspective esthétique fournit quelques pistes pour résoudre ce problème : proposer les mondes artistes comme modèle d'ouverture et de créativité pour enrichir les mondes singuliers (chapitre IV) introduire le jugement esthétique comme une autre manière d'envisager la composition d'un espace public plus ouvert aux singularités (chapitre V).

\footnotetext{
1 Nathalie Blanc est chercheure au laboratoire LADYSS (UMR 7533), Jacques Lolive est chercheur au laboratoire SET (UMR 5603).
} 


\section{Enrichir la problématique cosmopolitique dans une perspective esthétique}

Nous présenterons d'abord la problématique cosmopolitique; puis nos propositions pour enrichir cette problématique dans une perspective esthétique et enfin notre démarche de recherche.

La notion de cosmopolitique est élaborée par Kant en 1784 dans son Idée d'une histoire universelle au point de vue cosmopolitique ${ }^{2}$ (Kant 2002), et surtout en 1795 dans son Projet de paix perpétuelle (Kant 1999) pour garantir de façon durable la paix mondiale. Le projet cosmopolitique s'appuie sur trois hypothèses, le caractère pacifique des républiques, la vertu socialisatrice du commerce international et la fonction régulatrice de l'espace public international, pour proposer une République universelle, véritable fédération d'Etats libres et pacifiques, et une alliance des peuples garantie par l'émergence du droit international entre Etats et du droit cosmopolitique qui garantit les droits de l'étranger à l'égard de ces mêmes Etats (droit de visite et d'hospitalité). Cette anticipation d'une politique démocratique à l'usage des peuples du monde connaît actuellement un regain d'intérêt. Elle s'appuie sur la reconnaissance de l'interconnexion croissante des communautés politiques dans différents domaines incluant le social, l'économique et l'environnemental. Cette prise de conscience pourrait-elle devenir une culture cosmopolite partagée ? La plupart des réflexions contemporaines occidentales (notamment Held 1996, Archibugi Held 1999, Held 2002) portent sur les conditions requises pour une démocratie cosmopolite : une culture cosmopolite partagée ; un engagement préalable en faveur de la démocratie de la part de chaque citoyen du monde et la possibilité d'un ordre mondial politique qui dépasse les politiques étatiques traditionnelles et refuse la guerre des civilisations pour composer un monde commun pacifié avec les autres cultures et civilisations. Avec cette première conception des cosmopolitiques, la politique connaît un élargissement spatial. Elle devient politique du monde dans laquelle la notion de monde signifie « la surface de la terre où vivent les humains ${ }^{3}$ et par extension métonymique «la communauté des hommes vivant sur terre». Elle devient la politique planétaire qui s'adresse à l'humanité dans son ensemble, sans distinction de race, de pays, de culture... La cosmopolitique s'identifie à son objectif : la transformation de l'homme en citoyen du monde (Weltbürger).

Quelques chercheurs contemporains, (Beck 1986, Stengers 1997, Latour 1999, Descola 2005) vont encore plus loin dans cette exigence d'intégration cosmopolitique de l'étranger : selon eux, les cosmopolitiques supposent la composition d'un monde commun avec les «nonhumains » : les entités naturelles, les entités à risque, les artefacts scientifiques, les dispositifs techniques, les univers symboliques, etc. Il s'agit de «combler le fossé apparemment infranchissable séparant la science (chargée de comprendre la nature) et la politique (chargée

\footnotetext{
${ }^{2}$ Le titre allemand est Idee zu einer allgemeinen Geschichte in weltbürgerlicher Absicht. « in weltbürgerlicher Absicht» est traduit habituellement par «au point de vue cosmopolitique » ou «dans l'intention cosmopolitique », mais il signifie plus précisément «dans l'intention d'une citoyenneté du monde ». Les différentes significations du concept traduisent les évolutions qu'il a subies mais elles expriment aussi la pluralité des langues et le caractère intraduisible des principaux termes philosophiques. En l'occurrence et pour l'essentiel monde, Welt, kosmos. Par exemple, Kant utilise le terme allemand Welt mais pris dans un usage français, politique et «mondain », de monde. La reprise du terme par Latour et Stengers le déplace dans un sens environnemental de Umwelt (Uexküll 1956) tandis que nous souhaitons pour notre part l'enrichir des significations cosmologiques et cosmétiques attachées au nom grec ancien de kosmos. Sur ces distinctions, cf. l'article «Welt » in Cassin 2004, p. 1390-1396.

${ }^{3}$ Les différentes définitions du monde sont tirées du dictionnaire en ligne établi par le Centre National de la Recherche Scientifique et l'Université de Nancy 2 : le TLFI (Trésor de la Langue Française Informatisé) 2005.
} 
de régler la vie sociale), séparation dont les conséquences - affaire du sang contaminé, de l'amiante, de la vache folle - deviennent de plus en plus catastrophiques » (Latour 1999). Ainsi, la définition des cosmopolitiques varie avec celle du monde. Cette nouvelle conception des cosmopolitiques apparaît dans son contexte de crise environnementale et développement incontrôlé de la politique experte. Avec elle, la politique connaît un élargissement ontologique. Le monde ${ }^{4}$ y est considéré comme «l'ensemble de tout ce qui existe sur terre, perçu par l'homme et le plus souvent en opposition avec lui ». La politique du monde concerne d'autres êtres que les humains. Sa dimension planétaire lui est conférée par la crise écologique qui pèse désormais sur l'ensemble de la planète (changements climatiques globaux...).

C'est pourquoi, selon nous, il faut clairement distinguer les deux conceptions, les approches cosmopolites et les cosmopolitiques. Les politiques cosmopolites (Archibugi, Held) prennent en compte la diversité culturelle. C'est l'extension du multiculturalisme au plan mondial. En revanche, on parle de cosmopolitique (Stengers, Latour, Descola) quand l'étrangeté devient « irréductible », c'est-à-dire qu'elle dépasse la diversité culturelle et donne accès à des natures multiples. En effet, la notion de Nature ne reflète pas la diversité des pratiques scientifiques. Ce n'est qu'une idéologie moderne qui légitime les politiques expertes et technocratiques de tous ordres. Pour démocratiser les sciences et les techniques, selon Latour, il faut se débarrasser de la notion de Nature et étendre au domaine politique le modèle de la recherche scientifique qui progresse par controverses successives (Latour 2005).

Notre article prolonge cette seconde filiation tout en la complétant. Cette nouvelle cosmopolitique s'appuie sur le primat de la recherche : les experts et scientifiques sont les plus autorisés à parler de la nature, fût-elle devenue plurielle. Ils sont seuls autorisés à parler en son nom ; ils en sont les naturels représentants mais aussi, finalement, les seuls fondateurs. Ainsi, les savoirs indigènes sont-ils rabattus sur de la diversité culturelle. Nous souhaitons reprendre à notre compte l'ensemble des significations attachées au nom de cosmos (kosmos en grec antique) pour mieux faire entendre la richesse et le caractère intempestif des cosmopolitiques. Le cosmos est une variante du monde commun présentant trois caractéristiques principales : 1) la composition ordonnée et harmonieuse $e^{5}$ d'entités disparates (humains, choses, animaux, dieux) se situant hors de toute distinction moderne entre « nature » et «société », entre sujet et objet ; 2) le monde considéré comme une maison dans lequel les hommes trouvent leur place susceptible de conférer un sens à leur existence ; 3) les correspondances entre l'homme, défini comme un microcosme, et l'univers, le macrocosme dont il constitue le modèle réduit.

La réflexion cosmopolitique repense le monde commun comme un cosmos pour tenter d'intégrer l'étranger, d'absorber l'hétérogène dans un nouvel ordre du monde qui demeure juste et lui restitue sa beauté. Les cosmopolitiques explorent de nouvelles conditions de possibilités de la politique autour de trois aspects essentiels que confèrent aux cosmopolitiques la notion de cosmos : l'articulation microcosme / macrocosme dont témoigne le monde commun ; son habitabilité et son esthétique.

\footnotetext{
${ }^{4}$ La notion se rapproche alors de celle de Umwelt, le monde entourant, l'environnement.

5 «On pourrait rendre le terme kosmos par le syntagme baudelairien ordre et beauté». Chez les Grecs de l'antiquité, «le cosmétique participe à la définition du monde comme parure et joyau... La plus belle ordonnance c'est le monde... L'équivalence entre monde et ordre ne cesse de jouer ». Cf. Cassin 2004.
} 
Les cosmopolitiques, politiques du cosmos et politique des mondes, sont des politiques expérimentales qui tentent de composer un monde commun sans trancher les liens qui nous rattachent à nos mondes. La notion de monde met l'accent sur une compétence commune des sujets humains : nous fabriquons des mondes à travers un processus créatif qui nous constitue comme subjectivités : un sujet dans son monde qui l'englobe et le dépasse. Ces mondes du sujet pourraient constituer «les lieux du monde commun ${ }^{6}$. Fidèle à l'antique conception du cosmos comme articulation du microcosme et du macrocosme, une politique du cosmos tentera de composer entre eux nos mondes singuliers, «micromondes» en conflits, apparemment incompatibles, dans la visée d'un monde commun.

Le cosmos est un monde commun habitable, "l'habitat dans une maison du monde bien aménagée » (Sloterdijk 2003). Il suppose une partition entre un espace qui nous est propre, celui de nos mondes singuliers, et un extérieur étranger. Cette référence à l'habitabilité permet de mettre à l'épreuve les deux modes complémentaires de composition du monde commun privilégiés par la modernité, la justification politique et la preuve scientifique, qui vont homogénéiser à outrance les composants du futur monde commun, univers ou cité, et négliger la nécessaire partition au risque de rendre le «macromonde » inhabitable, en d'autres termes de détruire le cosmos. Cette décosmisation (Berque 2000) s'explique par le fait que les sciences et la politique composent trop vite en disqualifiant «l'esthétique au sens large, c'està-dire l'aisthêsis, la faculté de sentir de percevoir par les sens » (Berque 2000) ou, plus encore, la faculté d'imaginer et de se représenter comme visée un monde commun. Ces facultés combinent l'appréhension sensible et le jugement esthétique.

La perspective esthétique génère l'apparition d'une autre catégorie d'expérience que la scientifique (Dewey 1980, Reid, Taylor 2003). Elle ambitionne de créer un monde commun habitable, partant de la capacité de chacun et de tous collectivement à l'imaginer et le façonner ; c'est donc une perspective vitaliste et créative, parfois exubérante ; elle compose avec les affects et se veut plus rigoureuse à l'égard de la globalité du vivant. Elle donne une place aux artistes, nombreux à intervenir dans l'espace public, aux côtés des experts et des politiques. Elle pourrait faire contrepoids à l'hégémonie des faire-monde scientifique et technique.

Notre article s'attache à démontrer que la perspective esthétique constitue l'armature des cosmopolitiques ainsi reformulées. Nous développerons les arguments suivants. L'esthétique est au cœur du processus mondain car c'est la mise en forme des mondes singuliers qui permet leur appropriation par les habitants. Les mondes artistes constituent des modèles d'ouverture et de créativité pour nous garantir contre les clôtures (identitaires, territoriales) des microcosmes. L'esthétique constitue une modalité de passage entre les mondes singuliers et le monde commun. En particulier, le jugement esthétique peut revivifier l'espace public et organiser des débats plus respectueux des singularités.

Pour clarifier l'apport de la question esthétique aux cosmopolitiques, nous adopterons la démarche suivante. Il nous faut partir du monde des sujets ${ }^{7}$ tels que les analysent aujourd'hui recherches environnementales et politistes puis nous examinerons quelques pistes permettant de penser un monde commun à partir des ouvertures créatives proposées par les artistes.

\footnotetext{
${ }^{6}$ Nous transposons la formule d'Étienne Tassin qu'il appliquait aux républiques. Cf. Tassin 2003.

${ }^{7}$ Cette analyse des cosmopolitiques qui fait l'objet du second chapitre s'est nourrie des discussions avec Anne Tricot, géographe au SET et Didier Taverne, politiste à Montpellier III.
} 


\section{Les mondes singuliers : le sujet dans un monde plus qu'humain.}

La notion de monde est-elle pertinente dans l'optique cosmopolitique qui est la nôtre ? Comment se positionne-t-elle vis-à-vis des notions voisines d'environnement et de milieu ? Quels sont les objectifs de recherche qui nous feront la préférer ?

La notion de monde s'oppose à la notion classique d'environnement telle qu'elle est couramment utilisée en France ${ }^{8}$, puisque nous l'utilisons pour échapper au réductionnisme physico-chimique qui définit ce système de contraintes sur lequel l'homme agit et qui rétroagit sur l'homme. Il ne s'agit pas de nier l'existence des déterminismes à l'œuvre dans les écosystèmes mais d'insister sur les capacités d'initiative et d'invention dont font preuve ceux qui sont exposés à ces déterminismes. Nous refusons toute " politique de la Nature » qui légitime systématiquement l'expertise au détriment des savoirs et savoir-faire locaux. La seconde exigence, satisfaite par l'usage du monde, porte sur la relativisation, en fonction des sociétés, de la séparation de l'homme et de la nature. La notion de milieu, proposée par les géographes depuis Vidal de la Blache, y satisfait également puisqu'elle permet tout à la fois de considérer l'homme comme faisant partie intégrante de son environnement, tout en considérant ce dernier dans des rapports quotidiens et par rapport à chacune des sociétés considérées - tant aux niveaux idéels que matériels. Nous lui préférerons cependant ici la notion de monde parce qu'elle permet de valoriser l'expérience «d'environnementalisation » du sujet actif, impliqué, autonome et de «défataliser » franchement l'environnement fait, stabilisé par et pour l'étude des sciences physiques et de la nature. Les deux notions visent une même réalité mais alors que la notion de milieu insiste plus sur ce qui est donné, sur ce avec quoi l'action humaine doit composer, la notion de monde insiste plus sur ce que l'on fabrique, l'hybride socio-naturel. Le monde est ancré dans le subjectif, mais il incorpore des éléments du milieu. Le monde implique une perspective subjective et une visée collective puisqu'il faut la coopération d'acteurs multiples pour le créer. Enfin, le monde désigne une possibilité d'habiter ou sa mise à l'épreuve. Notre démarche s'inspire de l'approche phénoménologique (cf. la recension d'articles dans Seamon 2000), mais nous ne partageons pas son manque d'intérêt pour la politique.

Qu'est-ce qu'un monde pour le sujet et de quelle manière est-il comptable d'une dimension esthétique ? Pour cerner cette notion, nous suivrons quatre pistes exploratoires : qu'est-ce qu'habiter le monde ? Quelle place a l'esthétique dans la fabrication d'un monde qui nous dépasse ? Que devient le sujet dans ces conditions ? Comment évoluent ces mondes ?

\section{$\underline{\text { La partition du monde : le familier et l'inconnu }}$}

Fabriquer le monde est une opération complexe; la première piste d'élucidation que nous suivrons concerne l'habiter. Fabriquer le monde, c'est l'habiter (Bachelard 1957). «Elle (la maison) est le premier monde de l'être humain. Avant d'être "jeté au monde », comme le professent les métaphysiques rapides, l'homme est déposé dans le berceau de sa maison » Habiter le monde, c'est donc le partager entre un chez-soi et un extérieur ; c'est découper dans l'espace indifférent, un monde domestique, un lieu aménagé et approprié qui nous protège

\footnotetext{
${ }^{8}$ Dans la culture pragmatique des Anglo-saxons, l'environnement n'a pas cette signification puisqu'il manifeste « une réalité ouverte, interactive et évolutive, respectueuse de la spécificité et de l'autonomie du vivant». Cf. Charles 2001.
} 
(Bachelard 1957). Mon monde, c'est l'endosphère, une sorte de bulle (Sloterdijk 2002) qui fonctionne comme un prolongement de moi-même et me sert de camp de base pour explorer l'exosphère, le monde extérieur, incertain, inconnu, merveilleux et parfois hostile dans lequel je suis jeté. Ces territoires existentiels, véritables localités protectrices permettent aux sujets de construire leur autonomie, c'est-à-dire de s'inscrire dans la totalité en préservant leur différence (Roux 2002). Ces territoires localités protectrices sont aussi formées par une conception esthétique garante de leur insertion dans le monde. Dès lors, est non-lieu ce qui est dépourvu de signification esthétique. Deux types d'affirmations esthétiques : l'une publique tend à la monumentalisation des espaces, leur caractère intellectuel, froid ; l'autre privée confère par une série de signes invisibles son caractère significatif à l'espace.

\section{«Un monde, ça nous dépasse »}

La deuxième piste pour explorer le monde, c'est la notion de dépassement. Comment penser une fabrication dont le produit nous dépasse ? De quelle manière la question esthétique contribue t-elle à forger ce monde qui nous dépasse ? Dans la composition de son monde, le sujet incorpore des éléments (choses, paysages, animaux, humains, lieux) qui ont leur logique propre, qui le dépassent et sont rebelles et récalcitrants. La fabrication du monde est une tentative pragmatique qui peut échouer. L'homme configure son monde comme un projet, non pas un projet purement rationnel et cognitif, mais un projet baigné dans une tonalité affective (Heidegger 1986) et esthétique : les chasseurs de palombes transforment l'existant, ils organisent un monde finalisé par leur projet prédateur dont la pièce maîtresse est la fabrication de palombières, de dispositifs de capture. Ils développent collectivement une esthétique de la chasse qui incorpore lumières d'aube, odeurs de sous-bois, bruits d'ailes d'oiseaux. Les nourrisseurs d'animaux en ville reconfigurent la ville à l'aune de leur visée protectrice et de l'idée de ville vivante qui les anime ; cette esthétique urbaine fait référence au Paradis, et donc au jardin où toutes les espèces se côtoyaient. Un monde est ainsi défini comme une totalité d'objets instrumentaux et par la familiarité sensible avec une totalité de significations (Dardel 1990). Pour être réalisé, le projet de monde concerne ceux qui ont les mêmes attaches que le sujet; il doit aussi intéresser des alliés (Latour 1996) : concernés et alliés constitueront la matière humaine d'un espace communautaire, par exemple les chasseurs de palombes dans les espaces ruraux du Sud-Ouest. Comme tout projet, il peut échouer. Pour que le monde "prenne », il faut produire du concret, une concrescence (Whitehead 1929), un nouvel être-ensemble, une co-évolution du sujet et des composants essentiels du monde, un processus d'entre-capture (Deleuze Guattari, 1980, Stengers 199697) : la palombe doit se poser sur la palombière, le chasseur doit s'ensauvager (Heill 1994), devenir un homme «sauvage» qui aura le droit de tuer et l'habileté nécessaire... Les nourrisseurs produisent du décret facilitant leur gestion de ces animaux en contrepartie de quoi ils doivent les stériliser.

Ainsi, le monde produit dépasse et étonne. Il repose sur une série de procédés imaginatifs, sensibles et concrets qui l'oppose au rationnel, à la maîtrise, au dominé. Là encore, le sens apparaît mais dans ce rapport entre fabrication du monde et dépassement par le monde, entre immanence et quasi-transcendance (Barel 1984).

\section{La part des choses}

Cette relation intime du sujet avec son monde redéfinit la notion même de sujet. Le sujet n'est plus cet individu délimité par son enveloppe corporelle qui s'oppose aux objets. C'est qu'il 
existe la part manquante : les sujets envisagés sous l'angle cosmologique portent et participent du monde. Notre être s'étend aux choses ${ }^{9}$ qui nous concernent, par-delà notre limite corporelle. La saisie des choses par l'homme, la prédication (Berque 2000) est sélective, partielle, finalisée, sensible et symbolique à la fois. Elle oblige à fabriquer un monde satisfaisant du point de vue esthétique. C'est pourquoi plutôt que de s'inscrire dans un anthroposystème, la prédication des choses ouvre un monde. Par exemple, considérer la palombe comme une chose, ce n'est pas analyser le comportement d'un oiseau, mais étudier les conséquences de la saisie humaine de cet animal. La palombe-chose n'est pas un oiseau, c'est l'agrégation autour de cet oiseau : la palombière, l'homme sauvage, le droit de tuer, le repas en commun, la virilité, la beauté des lumières des sous-bois, etc. Ainsi les «palombes » rassemblent le collectif des chasseurs. Cependant, le monde n'est pas un réseau sociotechnique car les attachements à la palombe ne sont pas uniquement physiques, ils font place au symbolique, voire au surréel. On ne comprendrait pas la forme palombe sans la relation d'amour des chasseurs avec les palombes et la liberté qu'ils s'acquièrent en les tuant. Leur vie tourne autour d'elles : ils passent leurs week-ends à la palombière ; ils réparent et préparent la palombière pendant les vacances, etc. Les palombes sont le sens de leur vie : «ce autour de quoi tourne leur vie ». Leur rôle est si important qu'il suffirait sans doute de suivre les palombes dans leur migration - et les menaces qui pèsent sur elles - pour connaître les limites du monde des chasseurs concernés, sa configuration (la collection d'entités qui le constitue) et sa composition (l'articulation de ces entités). Les palombes seraient les « grands » de ce monde dont elles tracent les relations. Elles sortent grandies pour une autre raison : elles circulent dans différents mondes pour devenir la palombe des chasseurs, celle des écologistes et celle des gourmands. Du côté du cafard, le monde mis en branle est noir. On l'imagine technique : la bête circule par les tuyaux, se loge dans les fondations d'immeuble. Plus encore, noire elle-même, associée à la chaleur, elle représente l'étranger, l'autre et son visage sombre ; elle est emblématique d'une certaine pauvreté et redouble les catégories de l'exclusion. Ainsi, l'univers de cet insecte est réduit en pratique et riche en pensées. Dès lors, l'imagination, ce qui s'avère être la capacité à redoubler la présence de l'animal d'une symbolique et d'une esthétique, joue dans la participation au monde collectif (Blanc 2003) : le mode de figuration de cet animal est aussi celui du monde qui environne.

Cette capacité qu'a l'être humain de se couler dans les choses et de les façonner esthétiquement, par l'imagination et la sensibilité, contribue grandement aux subjectivités, aux modes de subjectivation cosmopolitiques : l'efficacité productive du monde procède du brassage d'hétérogène. La subjectivité est décollée du sujet. Il faut lui tracer une cartographie qui déborde les limites de l'individu (d'après Félix Guattari cité par Bourriaud 2001). La subjectivité se crée dans un va et vient entre un ici et maintenant vécu au présent et un là-bas et un futur projeté et imaginé. Les configurations mondaines combinent ainsi les attaches physiques et les attaches symboliques.

\section{$\underline{\text { La vie des mondes }}$}

Comment les mondes se maintiennent-ils tout en évoluant ? Nous formulons l'hypothèse que nos mondes s'apparentent à des systèmes vivants qui se transforment selon une dynamique de type auto-organisationnelle (Atlan 1979). L'idée est la suivante : les organismes vivants sont dotés d'un certain degré de complexité organisationnelle qui leur permet de résister au bruit,

\footnotetext{
${ }^{9}$ Sur la distinction entre objet et chose, cf. Lolive $2004:$ « Les choses et les objets ne se distinguent pas par leur essence, mais par leur modalité d'existence : l'objet est une chose stabilisée, maîtrisable, dont on peut négliger les conditions de fabrication et le tissu relationnel qui le socialise, mais cette stabilisation n'est que provisoire »
} 
c'est-à-dire aux perturbations survenues aléatoirement dans l'environnement, mais surtout d'assimiler, d'intégrer ces perturbations pour augmenter leur degré d'organisation, leur complexité. Pour pouvoir fonctionner ainsi, nos mondes seraient un mélange « de cristal et de fumée »(Atlan 1979). Dans notre exemple, le cristal correspondrait à la domesticité, l'organisation stratégique d'un espace de possession que l'on ouvre ou protège de l'inconnu merveilleux ou dangereux. Nos mondes sont des communautés (d'intérêts) unies par l'espace mais à n'être que cela, simplifiés et prédictibles, ils seraient d'une grande fragilité. Un des facteurs contribuant à la résilience des mondes est la capacité à produire de la métaphore. Le monde en image est un mode de pensée qui l'ancre esthétiquement et, à l'exemple du cristal, lui donne une grande cohérence formelle. En ce qui concerne cet animal esthétiquement sombre que représente la blatte, il n'est qu'à voir l'importance que prend la métaphore du « nid », et qui résiste à toute injonction scientifique, dans la représentation de son univers domestique. Dans ce cas là, le «nid» imaginé des blattes sous l'immeuble représente l'invasion potentielle, le grouillement animal et, se référant à l'étranger, constitue bien un discours permettant de penser sa présence en ces cités. Cette métaphore n'est pas positive, est même l'incarnation d'un racisme quotidien, mais permet de mieux comprendre les liens tissés entre monde naturel et social, entre bêtes et hommes dans un univers totalement symbolique. Deuxième point : pour se maintenir dans un environnement turbulent, pour rester ouvert et pouvoir évoluer, un monde doit être suffisamment complexe, en particulier il doit comporter également des zones informelles et «vaporeuses », en d'autres termes, nos mondes doivent être riches en redondance, en poches d'autonomie et d'incertitudes. Cette réserve de choses indéterminées, instables et inutiles constitue un atout pour créer de nouvelles opportunités et un gage d'adaptabilité en cas de perturbations externes (Soubeyran Barnier 1985). Mon propre monde inclut donc des éléments étranges et étrangers qui inquiètent et me dépassent. Cependant cette part incertaine de nos mondes joue un rôle essentiel dans leur renouvellement et leur expansion. Prenons deux exemples. D'abord certaines vies professionnelles (animation, recherche...) doivent apprivoiser l'incertitude et l'altérité sans les supprimer, puisqu'elles sont sources d'originalité et de renouvellement, ni s'y soumettre totalement, pour ne pas être qualifié de chimériques. Second exemple : la palombe est au cœur du monde des chasseurs concernés mais son comportement migrateur est préoccupant : d'où vient-elle, par où passe-t-elle, où repart-elle, reviendra-t-elle ? La chute rapide des effectifs évoque une possible disparition de l'espèce. Cette perspective pourrait bien pousser aux transformations du monde des chasseurs de palombes du Sud-Ouest dans le sens d'une expansion du collectif. À l'inverse la prolifération animale, l'idée même d'espèces invasives, inquiète profondément. Que ce soit les fédérations de chasseurs ou les gestionnaires de milieux tous sont demandeurs d'une métrologie propre. La modification des modes de gestion nécessiterait des procédures de consultation des chasseurs entre eux et une réévaluation des traditions.

À l'issue de ce second chapitre, il convient de souligner l'intérêt d'une analyse des mondes singuliers pour mieux traiter la crise environnementale. La crise environnementale réside également dans une incapacité à accueillir et à vivre avec l'étranger, en particulier les entités environnementales. Dans notre incapacité à considérer ce qui n'est pas nous. Une cosmopolitique bâtie autour de l'étranger-étrangeté permet d'y faire face. Parce qu'ils expriment l'inclusion des sujets dans un monde plus qu'humain, les mondes singuliers nous aident à réactiver une conception sensible de l'écologie. Comme l'indique Patrick Howard (Howard 2005) :

«Les idéaux épistémologiques de clarté, détachement et objectivité ont rendu silencieuse la voix de la Nature. Depuis plus de 300 ans, nous avons tenté de séparer nos personnes des processus organiques et des rythmes du monde naturel. Malheureusement, nos enfants ont appris leurs leçons. Nous avons besoin d'une 
autre manière de connaître nos lieux et le monde, Comment réactiver les articulations et les relations complexes des choses... La biorégion et un engagement corporel imaginatif et participatif avec les autres qu'humains...»

Mais il subsiste une question: comment s'effectuera la mise en politique de ces petits mondes, qu'ils soient artistes ou pas ? Comment les intégrer dans notre monde commun ?

\section{La difficile prise en compte des mondes singuliers dans notre monde commun}

Les clôtures qui limitent parfois la capacité créatrice des mondes singuliers et les réductions dont ils sont l'objet dans les débats politiques et les expertises nous obligent à imaginer de nouvelles règles de composition pour les intégrer dans notre monde commun.

\section{Clôtures cosmologiques et violence}

Quelles sont les menaces qui s'attachent aux mondes ? Hannah Arendt (Arendt 1951) a cerné les pathologies de l'idée avec sa notion d'idéologie, la logique d'une idée à laquelle le monde est censé se plier. Ce modèle permet de cerner les pathologies des mondes en redéfinissant la notion de cosmologie (Stengers 1996-97) comme la logique d'un monde microcosmique à laquelle le monde «commun » est censé se plier ${ }^{10}$. Le microcosme protège une communauté du changement en stabilisant l'environnement (Sloterdijk 2000) il tend ainsi à l'immuniser contre l'événement, l'intrusion de l'inattendu, la capacité de commencer. Ce faisant, les clôtures cosmologiques entretiennent et renforcent l'incommensurabilité entre nos mondes et l'impossibilité du consensus par échange d'arguments. L'ouverture des mondes singuliers ne va pas de soi. Les attaches à la nature et au proche (lieux, pays, etc.), ces attachements de proximité, soutiennent des implications personnelles fortes et leur mise en péril suscite une émotion intense. Il en résulte parfois une violence réactive qui sanctionne les atteintes au monde et aux relations avec lui. Une partie des excès du mouvement des chasseurs lui est peut-être imputable.

\section{$\underline{\text { Singularités et réductionnismes }}$}

Si les mondes singuliers sont parfois inassimilables, du fait de leur singularité extrême, ou s'ils refusent parfois de s'ouvrir au politique et à l'événement, un comportement symétrique est constaté chez les tenants des modalités usuelles de fabrication de l'intérêt général qui réduisent les singularités en sacrifiant les attaches constitutives du sujet pour en faire un citoyen désintéressé, abstrait, détaché, un pur esprit. Comment justifier ces interventions de l'habitant, du riverain, de l'amateur de paysage et comment les sauver de la disqualification opérée au nom des conceptions classiques de l'intérêt général ? Malgré tout leur intérêt, les propositions de démocratisation de l'expertise proposées par certains de nos collègues (Latour 1999, Callon, Lascoumes, Barthe 2001) ne semblent pas constituer une solution suffisante puisqu'elles mettent trop l'accent sur les compétences publiques et les compétences expertes du sujet, fût-il profane, en disqualifiant d'autres compétences du sujet, en particulier ses compétences territoriales, celles qui concernent l'intime, le parcours de vie, et surtout

\footnotetext{
${ }^{10}$ La comparaison avec la notion arendtienne s'arrête là car les mouvements totalitaires ont jeté leur dévolu sur les idéologies au $\mathrm{XX}^{\mathrm{e}}$ siècle mais il n'y a aucune fatalité de ce type concernant les cosmologies.
} 
l'esthétique au sens classique, c'est-à-dire l'aisthêsis dont nous avons déjà parlé. Ces compétences qui combinent l'appréhension sensible et l'imagination s'exercent à travers l'expérience et le jugement esthétique.

\section{$\underline{\text { La perspective esthétique pour respecter les contraintes cosmopolitiques }}$}

Dès lors, les contraintes cosmopolitiques se précisent : 1) comment imaginer un monde commun habitable car respectueux et incitatif d'une pluralité des mondes singuliers ? 2) à quelles conditions peut-on dépasser les perspectives locales, voire les clôtures des dits mondes pour autoriser les cheminements créatifs ? La perspective esthétique nous semble seule capable de lever ces contraintes. Concernant la contrainte d'ouverture, les possibilités créatrices mises en œuvre dans les pratiques artistiques proposent un modèle de sujet actif sous ce registre ; les principes de composition des mondes artistes en garantissent l'ouverture et l'indétermination. Ils en garantissent aussi la richesse voire la densité. Par ailleurs, les mondes communs esthétiques demandent également leur place dans l'espace public. Sur le plan esthétique, la conception kantienne du jugement élaborée à partir de l'esthétique satisfait la contrainte de pluralité. La perspective esthétique d'Arendt satisfait la montée en généralité et la nécessité d'une action vive, globale à l'égard du politique. Cependant, l'incorporation de l'esthétique dans le champ des cosmopolitiques oblige à approfondir la création individuelle et les modes désintéressés d'investissement de l'espace public. Le jugement esthétique est d'ailleurs pour Kant : « le libre jeu » harmonieux de l'imagination et de l'entendement (Kant 1993, §35). Elle oblige aussi à comprendre la place de l'art dans le champ social et politique : perspective toujours renégociée qui donne la mesure du bouleversement à l'œuvre dans le champ social et politique, qui implique une écologie politique ${ }^{11}$.

\section{Espaces publics et monde commun}

Comment traiter ces questions de la politisation des mondes et de l'esthétisation de la politique ? Une des principales modalités de politisation, c'est la mise en débats dans l'espace public. Que faut-il entendre par espace public ? La notion d'espace public semble écartelée entre, d'une part, une préoccupation d'aménagement très pratique et concrète de places, jardins ou rues, et d'autre part, une recherche très théorique de fondements pour une vie politique démocratique. Ce caractère double de l'espace public est présent dès l'Antiquité : il n'existe pas d'espaces publics sans agora, sans forum, sans Polis qui constituent des lieux de mémoire des actions des citoyens, qui les immortalisent. Il n'existe pas de principes qui ne s'incarnent dans des corps, des biens ou des lieux. Cette ambivalence fait la richesse du concept et va justifier l'utilisation que nous en ferons. On peut considérer l'espace public comme un modèle réduit, un paradigme au sens grec antique, du monde commun. Les règles de fonctionnement de l'espace public renvoient aux règles de composition désirées du monde commun. L'espace public est la mise en politique du monde commun, mais cette mise en politique n'est pas qu'une mise en débat, c'est une mise en acte voire une concrétisation car l'espace public est également un espace de vie. De lieu du débat qu'il était, l'espace public devient l'enjeu du débat.

\footnotetext{
${ }^{11}$ En effet, ces exigences cosmopolitiques renvoient à un contexte de crise environnementale caractérisée notamment par une globalisation des enjeux qui va de pair avec la réhabilitation des localités et riverainetés menacées par les grands projets et les difficultés d'un environnementalisme plus préoccupé de technique, de savoir-faire, que du sens global de son action ; un état de droit dont la fragilité va de pair avec l'ampleur des dégâts écologiques et la faiblesse des solutions confronté aux menaces d'un développement et d'une exploitation des ressources naturelles souvent violente.
} 
C'est pourquoi nous formulerons nos hypothèses sur la démocratisation des espaces publics par l'esthétique en nous appuyant sur des tendances actuellement à l'œuvre. Les œuvres et les interventions d'artistes se multiplient dans l'espace public et des projets de développement local à composante culturelle, artistique y prennent une place croissante. On assiste à une évolution récente des contestations associatives qui mobilisent des critères esthétiques pour légitimer les conflits (luttes paysagères, expérimentations urbaines) qu'ils animent. Cette importance de l'esthétique dans l'espace public suscite des interrogations sur : 1) la place et le rôle des artistes dans l'espace public ; 2) la possibilité d'utiliser des critères esthétiques dans l'espace public. C'est ce que nous allons discuter à présent.

\section{Les mondes artistes : un modèle d'ouverture et de créativité pour les mondes singuliers}

Les mondes artistes nous apparaissent comme un modèle d'ouverture pour les mondes singuliers. Il faut se tourner vers la création vive, contemporaine, qui génère une perspective esthétique différente (Jimenez 2004). Les artistes, aujourd'hui très présents dans l'espace public et y disposant d'une liberté créatrice plus grande que les urbanistes, sont associés une esthétique qui donne une place au contexte politique, social ou plus formel (Bourriaud 2000). Par ailleurs, l'art contemporain fait une place inédite au spectateur qui le rend artiste à son tour. Cette collusion de l'artiste et du spectateur qui fait l'objet d'une esthétique ainsi que le rôle dévolu récemment à l'artiste comme animateur de l'espace public rend pertinent de conduire notre réflexion sur la place de question esthétique dans les cosmopolitiques en partant des mondes artistes. Une grille de lecture, à mi-chemin entre observations empiriques et lectures théoriques, permet de voir les règles de compositions des mondes artistes dans la perspective d'un monde commun; ainsi que les modes d'intervention dans l'espace public. Ainsi précise-t-on les ouvertures créatives et les exigences éthiques que permet la composition esthétique du monde commun : respiration, gestation, respect et densité. Ainsi également comprend t-on en quels termes décrire les contributions singulières à un espace public doté de nature, de sensibilité, d'imagination bref à un espace public, modèle en quelque sorte de celui que devrait nous présenter toute réflexion sur les cosmopolitiques. Un espace public riche. Un espace que le jugement esthétique alimente.

Ces concepts sont importants également pour une cosmopolitique soucieuse de l'intégration d'une pluralité de mondes et de leur richesse maximum. Ainsi les artistes fournissant des images aux mots : "les concepts doivent, pour trouver leur efficace, revêtir les atours de la réalité concrète, se territorialiser sur des images » (Félix Guattari cité par Bourriaud, 2001). Ils nous fournissent des exempla pour exercer notre jugement politique et imaginer le devenir cosmique d'un monde commun.

\section{$\underline{\text { Respiration }}$}

La notion de respiration ${ }^{12}$ (Tassin 2003 d'après Arendt 1958, Lolive in Berdoulay et al. dir. 2004) permet de «défataliser» l'environnement et de réanimer la politique «morte », institutionnalisée, pour leur restituer une dimension expérimentale et pragmatique. Elle redéfinit la démocratie comme une pulsation entre des séquences d'innovation marquées par

\footnotetext{
${ }^{12}$ C'est le philosophe Étienne Tassin qui a le premier proposé cette notion de respiration pour distinguer chez Hannah Arendt le monde fabriqué par l'artifice humain et l'espace déployé par les actions des hommes.
} 
l'action politique et des séquences de stabilisation institutionnelle et socio-technique (Latour 2004) où la politique vive se coagule, se cristallise. La création artistique participe de cette respiration. La notion est précisée lors de l'entretien avec l'artiste paysagiste MP qui réalise des jardins éphémères dans des territoires urbains délaissés. Il nous indique deux modalités de l'action artistique qui concourent à la respiration. La première, c'est fluidifier : "Pour contourner la règle, il fallait changer de casquette. Impossible d'intervenir en tant que paysagiste, il fallait intervenir en tant qu'artiste». Cette intervention est, d'une certaine manière, le refus de l'arrêt : "C'est simple, je me déplace en permanence. La création est un des éléments de transformation du monde. On invente tout en permanence... » Dans un espace urbain français, saturé d'institutions, de règlements et de culture politique, l'artiste est autorisé à contourner la règle. La population admet cette transgression et la considère comme normale venant d'un artiste. Les politiques et les administrations lui délèguent également ce pouvoir de transgression. L'émancipation n'est pas totale, la transgression est partielle, elle s'effectue dans des interstices (nous y reviendrons plus loin). La seconde modalité, c'est potentialiser : «Nous intervenons dans des non-lieux, des espaces interstitiels pour révéler des potentialités inexploitées, inexplorées dans la ville ». Le lieu choisi pour implanter le "Jardin sauvage », situé en contrebas de l'avenue du Président Wilson, encaissé et à l'ombre est un lieu oublié, effacé des mémoires, voire un non-lieu. En effet, il n'existait pas puisqu'il était invisible avant la construction des passerelles d'accès au restaurant du Palais de Tokyo. Le résiduel, le rebut recèle des potentialités inexploitées. La potentialisation s'apparente ici au recyclage, voire à l'alchimie puisqu'elle permet de transformer des déchets en valeurs, de changer le plomb, vil métal, en or. La notion d'interstices constitue le troisième aspect révélé lors de l'entretien. La création se produit dans les interstices que les structures autorisent, rendent possibles, tolèrent (Whitehead, 1929). Pour les penseurs de l'art contemporain, l'œuvre d'art représente un interstice social (Bourriaud 2001): "C'est un espace de relations humaines qui, tout en s'insérant plus ou moins harmonieusement et ouvertement dans le système global, suggère d'autres possibilités d'échanges que celles qui sont en vigueur dans ce système... L'œuvre d'art se présente comme un interstice social à l'intérieur de laquelle ces expériences, ces nouvelles "possibilités de vie » s'avèrent possibles... \C'est dans ces interstices, c'est-à-dire dans les zones informelles, que se produisent les événements "esthétiques » qui sont parmi les plus créatifs. Les événements esthétiques qui se produisent dans ces régions privilégiées du monde commun sont les garanties que ce monde reste commun, c'est-à-dire ouvert, susceptible d'accueillir les autres qui sont toujours inattendus.

\section{Gestation}

L'image de la gestation est formulée par la peintre $\mathrm{KB}$ qui réalise des tableaux lumineux à partir de traces photographiques qu'elle éclaire de différentes manières : " ma démarche, c'est d'essayer de comprendre comment les flux (notamment lumineux) nous arrivent à travers la matière et nous permettent de voir l'espace». Inquiète par la globalisation qu'elle analyse comme le «progrès » d'une humanité qui abrase les différences, KB refuse cet avenir imposé par la société. Elle s'y oppose en tant que sujet ouvert aux flux du cosmos : "La réalité, le monde, la perception du monde que j'ai est un tout extrêmement lié, organique. Quand je vois un arbre, je ne pense pas un arbre, je pense à la matière, aux flux qui arrivent et la traversent... Je me suis intéressé au mot latin per-spicere qui signifie voir à travers. De là, j'ai retravaillé l'idée de perspective... Je suis très sensible à la lumière... Je suis partie de l'idée d'une visualisation interne de mon propre organisme avant de passer au cosmos...». Ce refus de la globalisation au nom du sujet inséré dans le cosmos - et de la relation entre microcosme et macrocosme - fait référence implicite aux cosmopolitiques. Cependant la prise en compte de l'hétérogénéité du cosmos et l'importance des liens faibles en son sein lui 
font refuser la notion d'un ordre cosmopolitique : "C'est tellement lié, ça grouille tellement de choses infimes de partout, que l'idée de vouloir mettre de l'ordre, c'est couper tout lien... Kawamata (un artiste qu'elle aime bien) rend visibles les liens invisibles, avec des éléments d'une fragilité extrême, qui lient les espaces entre eux... Ces éléments montrent à quel point les choses sont imbriquées... Cela rejoint ce que je te disais par rapport à la gestation : si tout agit sur tout, tu ne peux pas diriger aucun flux de manière précise. Ce serait couper une partie de l'univers... » Dès lors, elle préfère se placer «du côté de la gestation », une posture qui combine le devenir (Whitehead 1929) avec la prise en compte de la fragilité des liens évoquée précédemment. "Il émane de mon travail un univers en devenir, qui s'apparente à une conception du monde non encore arrivée à terme, mais se développant sous des formes déjà existantes dans l'espace. C'est comme si le liant d'un monde (d'un informe) organique mettait çà et là en lumière une totalité pressentie, prête à éclore dans le regard de l'autre. Je tiens à préciser que les formes que je travaille ne s'inspirent pas à proprement parler de cette image foxtale. Un tel rapprochement vient probablement de ces formes que je vais chercher sur une source lumineuse, et donc à l'origine...» Cette réflexion nous invite à relativiser deux conceptions dominantes des cosmopolitiques : l'universion ${ }^{13}$ (Berque 2000) du cosmos par les sciences (physiques et de la nature) et la souveraineté d'un Léviathan mondial. Le monde commun n'est pas le produit d'une composition rapide, volontaire et réductrice, c'est plutôt une visée, une sorte d'idéal régulateur qui doit inspirer les actions et les politiques. Les cosmopolitiques impulsent un processus de recosmisation du politique car elles sont animées simultanément par le souci du monde commun et le respect des mondes singuliers : ces complexités de lumière, de temps, de matière où vivent les gens, les récits et les personnages. Comme le monde commun est un processus inachevé, pluriel et ouvert, les cosmopolitiques n'ont pas vocation à remplacer l'ensemble des politiques, leur champ d'application concerne surtout les zones de frictions entre les mondes singuliers, là où se posent des problèmes aigus de coexistence intermonde (controverse, menace de guerre, de disparition). Comme le(s) monde(s) n'est ni appropriable, ni maîtrisable, les cosmopolitiques sont peu compatibles avec les théories classiques de l'action qui conjuguent rationalité, volontarisme et linéarité. Il faudrait ébaucher des théories alternatives de l'action : épouser la propension des choses, assister le naturel, sélectionner les contraintes pertinentes et y préparer l'acteur humain, prendre en compte la résistance de l'objet, l'action est transformation, etc. (Jullien 1996).

\section{$\underline{\text { Respect }}$}

La troisième image qui associe respect, cadrage, bonne focale et proximité des choses est formulée par le photographe SA, photographe documentaire et enseignant dans une école d'urbanisme. "La photographie, c'est un miroir déformant... Le document, c'est la prise de conscience qu'un $50 \mathrm{~mm}$, par exemple, est plus proche de votre oeil, en termes de focal... Finalement, être proche des choses, c'est aussi les respecter. Un format est souvent lié à l'objet que l'on est en train de photographier. Respecter une maison, c'est l'encadrer dans un format qui respecte ses proportions... Il s'agit de ne pas toucher aux choses, de mettre une distance... La question du sujet est très importante, mais elle est évacuée rapidement, on est dans le sujet, c'est-à-dire on est sur le territoire désigné, tu nous montres des choses qui posent problème etc. OK, maintenant on ne va parler que de la forme et, du coup, on a une espèce d'adéquation entre un sujet qui a besoin d'avoir le support d'une forme et un territoire...» L'image proposée par notre interlocuteur appelle une posture éthique, une

\footnotetext{
${ }^{13}$ Augustin Berque définit l'universion comme la revendication d'universel proposé par les sciences physiques et de la nature : cette forme d'unicité réduit le divers à l'un et la réalité à une version unique. Elle suppose un coup de force envers le monde sensible.
} 
morale en situation toute imprégnée d'esthétique. D'abord, l'image pointe la relation entre le format de la photographie et l'objet photographié, ici une maison. La maison convoque un cadre qui respecte ses proportions. Si nous laissons filer la métaphore, nous dirons que les territoires existentiels produits par les sujets et populations concernés constituent la seule échelle pertinente des cosmopolitiques et qu'ils ne sont pas réductibles à la dichotomie entre le local et le global (Berdoulay, Entrikin 1998). Être proche des choses, mais ne pas les toucher : cela évoque pour nous ce choix du monde singulier comme référentiel d'action, mais sans en adopter la clôture. La deuxième proposition «cosmoéthique » est la relation d'adéquation entre le « sujet » et la forme. Le « sujet» - le territoire désigné avec les choses en son sein qui posent problème - a besoin d'avoir le support d'une forme. La forme permet la rencontre, le dialogue autour du problème. Si l'on considère la forme artistique comme une modélisation du monde singulier, cette forme-monde devient le support éthique : "La forme devient visage. Produire une forme, c'est inventer des rencontres possibles; recevoir une forme, c'est créer les conditions d'un échange » (Bourriaud 2001). On se rappelle que pour le philosophe Emmanuel Levinas, le visage représente le signe de l'interdit éthique, c'est ce qui m'ordonne de servir autrui et ce qui m'interdit de tuer (Levinas 1982). Transposées aux formes-mondes, ces exigences s'apparentent à celles de la biodiversité : chaque monde est une création singulière, originale qu'il faut respecter et protéger (cosmodiversité) tant qu'elle ne détruit pas les autres territoires existentiels. Le monde singulier est aussi ce dont un groupe est responsable (accountability). Ce groupe certes manque de maîtrise, mais il reste responsable de ce qu'il a incorporé dans son monde et qui va co-évoluer avec lui. Avec la posture modeste de $\mathrm{SA}$, nous avons affaire à une éthique située où la convenance (Thévenot 1990) et la justesse (Boltanski 1990) rejoignent les préoccupations esthétiques.

\section{Densité}

L'image de la densité revient sous la plume de certains critiques de l'art contemporain sans qu'ils la problématisent : "L'esthétique crée des modus vivendi permettant des rapports sociaux plus justes, des modes de vie plus denses, des combinaisons d'existence multiples et fécondes... Une cuvre dotée d'une forme définie, d'une certaine densité... (Bourriaud 2001). Avec le passage de l'artiste au médiateur, promu par les tenants de l'esthétique relationnelle, comme le critique d'art Nicolas Bourriaud, si l'on ne produit plus d'objets, où est le beau ? Comment retravailler cette question de la beauté saturée d'informations, de théories, d'avis autorisés ? Une question voisine plus accessible apparaît dès lors, celle de la densité. Comme pour la question de la liberté, on y retrouve un critère de jugement, d'évaluation. La notion de densité (peut-être une notion plus satisfaisante que celles de profondeur, d'épaisseur...) traduit le moment où les différentes choses qui sont présentes n'apparaissent plus pour elles-mêmes. Elles sont dans une unité. Elle évoque la Phusis, le jaillissement, l'élan quasi-naturel car le nombre des relations n'est pas visible. La notion s'oppose à celle de platitude, celle des réseaux socio-techniques et de la société de la sociologie en général. Elle signifie le caractère épais, multidimensionnel des relations, la richesse des liens entre le sujet et son milieu. Ainsi l'art, ou la préoccupation du sensible en ville, invite à tisser de nouveaux liens entre l'habitant et son espace d'habitation : la ville fonctionne d'ailleurs parfois comme une métaphore de tout espace public. Ces liens sont multiples, complexes, épais et pas seulement fonctionnels. La densité permet de qualifier le devenir cosmos des mondes singuliers pour contrebalancer une conception trop fabricatrice, exhaustive et anthropocentrée de ceux-ci. Il existe une relation entre densité et monde quand celui-ci tend vers un cosmos. A contrario, la preuve par certaines créations contemporaines qui font exister une société avec ses objets proliférants, insignifiants et dérisoires. Mais ce 
n'est pas un monde, il est trop horizontal. Ces créations dérivent trop des procédés. Un monde, c'est quelque chose qui nous dépasse, quelque chose qui nous étonne.

À suivre ces artistes, la notion d'ouverture se précise peu à peu. Le monde ouvert n'est pas un simple réservoir de possibles. Cela sous-entendrait une conception instrumentale et finalement trop maîtrisée de l'action conçue comme utilisation stratégique et novatrice d'un potentiel disponible «sous la main ». Pour enrichir la notion, on peut se référer à Arendt qui fait du politique un registre de l'action ouvert à la pluralité des récits dont on ne peut prédire ni la finalité, ni les fins, mais la philosophe ne précise pas les modalités d'une action politique de ce type. Le travail de certains artistes nous fournira d'autres indications. Il peut être décrit comme la construction d'un cheminement par lequel l'artiste accompagne l'œuvre. Cette conception revalorise l'empirique qui devient une théorie en acte, tissée entre le corps de l'individu qui la confectionne et un corps de savoir-faire qui a capacité d'engendrer l'action. Ainsi, le monde ouvert des artistes n'est pas un chaos, c'est quelque chose qui préserve des cheminements possibles; ce n'est pas rien, mais un lieu où des choses prennent forme. Les quatre images (respiration, gestation, respect, densité) analysées nous font voir les principes de composition des mondes artistes et elles nous fournissent des indications pour penser une cosmoéthique (Stengers 1996-97), une morale du monde commun.

Ce travail sur les mondes artistes contemporains demande un approfondissement sur plusieurs points. La forme finale du travail pictural, photographique ou d'installation etc. des artistes témoigne d'un processus. Ce processus engage, a minima : une autonomie, ou la construction singulière de référents qui aient force de loi pour l'artiste, la capacité à instruire une métaphore et un jugement esthétique qui en établisse la pertinence dans l'espace public. Le travail de l'artiste n'est pas uniquement de l'ordre de la représentation ; la place du jugement esthétique dans l'élaboration d'une œuvre peut être considéré comme un fait contemporain ; l'artiste élabore son œuvre en fonction d'une jugement qui fait de l'esthétique une condition de formalisation d'un débat démocratique. En effet, ces divers témoignages mettent en évidence la pluralité (ou l'hétéronomie) des approches artistiques, mais leur « contextualisation » montre une montée en généralité, formelle et rhétorique, associée à une réflexion sur l'espace public. Ce jugement est un risque, un critère de discernement et s'offre comme l'une des possibilités de respiration dans un monde figé, institutionnalisé et régi par le droit. Il représente une des sources de création et d'innovation. Outre cela l'implication croissante de l'artiste dans l'espace public correspond à l'élargissement de la notion d'art et d'artiste et à la dilution de l'esthétique contemporaine. Elle met en jeu, alors, un artiste devenu habitant, dont l'œuvre repose sur l'esthétisation de l'espace public, et un habitant sommé de venir artiste, et de mettre en œuvre des capacités de jugement esthétique. Ainsi notre proposition d'utiliser l'expression de «jugement esthétique » pour définir ce travail d'objectivation va avec la définition que propose Kant, le travail de relecture qu'opère Arendt et les réflexions plus contemporaines.

\section{Un espace public esthétique valorisant la pluralité des formes}

L'autre apport de la perspective esthétique réside donc dans le jugement esthétique qui permet de revivifier l'espace public englué dans une confrontation-connivence entre les références à l'intérêt général et la montée des enjeux d'expertise. Nous analyserons dans cet article la pertinence théorique de ce rapprochement en réservant l'analyse des mobilisations associatives centrées sur le jugement esthétique pour un autre article (Lolive 2005). Le jugement esthétique naît au $18^{\text {ème }}$ siècle notamment avec Kant. Des philosophes parmi 
lesquels Hannah Arendt ont travaillé ce jugement, sa forme ainsi que la place qu'il prend dans l'espace public. Notre réflexion reprend donc certains des termes du jugement esthétique formulé par Kant, et de l'interprétation qu'en fait Arendt. Cependant nous prolongeons le débat.

\section{Le jugement esthétique respecte la singularité et la pluralité des mondes}

La pluralité des petits mondes, la richesse singulière de certains d'entre eux, l'importance qu'ils revêtent pour ceux qui les habitent et les imaginent nous inspirent du respect mais cela ne suffit pas. Comment leur rendre pleinement justice et leur accorder une place dans notre monde commun ? Ni les explications scientifiques, ni les mécanismes de l'intérêt général ne le peuvent. Neutralité axiologique, réductionnisme et justification vont saccager ces mondes fragiles et complexes, pétris d'imaginaire, de valeurs et d'affects dont pourtant la figuration artistique parvient à rendre compte. D'ailleurs, c'est en réfléchissant sur la perception esthétique dans sa Critique de la faculté de juger (Kant 1993) que Kant ${ }^{14}$ a élaboré une théorie du jugement qui permet de « fonder en raison ${ }^{15}$ l'importance de ces petits mondes.

Pour Kant, la perception esthétique n'est pas seconde par rapport à la théorie ou à l'action pratique, elle offre au sujet le plaisir et les avantages d'une contemplation désintéressée. En effet, la contemplation esthétique ne tente ni de saisir l'objet au travers d'une grille conceptuelle, ni de l'instrumentaliser par rapport à des objectifs pratiques précis : « le sujet ne réduit pas l'objet de sa perception à certaines de ses caractéristiques, mais le perçoit en sa plénitude non représentable... dans la présence de son apparaître... L'apparaître esthétique dont il est question ici n'est pas une simple opinion subjective... Il s'agit plutôt d'une façon particulière dont les phénomènes se présentent et qui peuvent être compris sur un plan intersubjectif »(Seel 2004). Dès lors, on voit apparaître la possibilité d'un espace public esthétique.

Kant définit le jugement esthétique, ou jugement réfléchissant, comme la faculté de juger qui traite du particulier quand on ne dispose pas d'un universel - règle, principe, loi, norme pour le subsumer. Comment faire ? Kant propose alors deux solutions (Arendt 1991, 13 conférence). La première, c'est l'idée de finalité. « Tout objet, écrit Kant, en tant qu'il est un particulier qui requiert et contient en lui-même le fondement de sa réalité actuelle, a une fin » (Arendt, idem). Mais quelle est la fin des objets d'art? «Ils ont pour «fin» de plaire aux hommes de faire en sorte qu'ils se sentent chez eux dans le monde. On ne peut jamais le démontrer, mais la finalité est une idée d'après laquelle on régule les réflexions des jugements » (ibidem). «Le jugement réfléchissant cherche et trouve son principe dans la réflexion qui dégage de la finalité de la forme, la forme de la finalité » (Kant 1993 cité par de Duve 1989). L'autre solution est celle de la validité exemplaire définie et utilisée par la rhétorique. On cherche un particulier que l'on juge le meilleur possible sous le rapport de l'essence ou de la qualité étudiée. Achille, le guerrier de l'Iliade, est un exemple de guerrier courageux. Cet «exemplaire restera un particulier qui dans sa singularité même exhibe la généralité qu'on ne pourrait déterminer autrement. Le courage est comme Achille » (Arendt id.).

\footnotetext{
${ }^{14}$ Kant est le fondateur de la notion de cosmopolitiques (Kant 2002) et il est normal que nous le croisions dans notre réflexion. Nos analyses du jugement esthétique s'inspirent aussi des réflexions de théoriciens de l'esthétique (de Duve 1989, Seel 1998, 2004) qui réfléchissent sur sa troisième critique (Kant 1993) et d'Hannah Arendt qui pense les relations entre le jugement esthétique et la politique (Arendt 1991).

${ }^{15}$ Nous reprenons là une expression chère à Olivier Soubeyran, géographe à l'Université de Grenoble, avec qui Jacques Lolive a organisé le colloque de Cerisy consacré aux cosmopolitiques.
} 
En tant que réfléchissant, le jugement esthétique est un principe actif de cosmopolitiques dans la mesure où il permet d'apprécier les mondes construits, leur cohérence, ainsi que leur place dans un espace public ou monde commun. Dès lors, la forme fonctionne comme indicateur plus que la connaissance issue de la rationalité scientifique. Cette forme (en tant qu'indicateur) donne sens à l'action politique ; elle la réfléchit. On peut prolonger les analyses d'Arendt pour qui la politique a la forme de l'espace public. Cette forme «espace public » est la finalité du politique et l'inverse est également vrai ; du coup la forme devient un moyen régulateur, la représentation est régulatrice. Avec ces notions de validité exemplaire et de jugement désintéressé, on comprend mieux pourquoi l'expérience esthétique peut nous offrir un modèle de vie. Dans cette disponibilité respectueuse au réel, nous faisons l'expérience de la richesse et de l'ouverture du monde qui nous offre un espace de jeu pour nos actions et nos réflexions. L'œuvre d'art nous place dans un rapport particulier au monde et à nous-mêmes : intensification des sens, distanciation par rapport à l'intuition (Seel 1998).

\section{Proximité du jugement esthétique et du jugement politique chez Arendt}

Dans ses Conférences sur la philosophie politique de Kant ${ }^{16}$, Hannah Arendt se réfère à cette conception kantienne du jugement esthétique, c'est-à-dire la faculté de juger pour traiter du particulier, pour définir le jugement politique ${ }^{17}$. Arendt s'empare de Kant pour satisfaire une exigence vitale de résistance et de reconstruction. Après la chute des totalitarismes, elle s'interroge : à quelles conditions peut-on rouvrir un monde commun ? Dans ces réflexions de la dernière partie de sa vie, Arendt met l'accent sur l'aptitude à juger qui permet de résister au mal et à la désolation de l'homme en système totalitaire. Deux questions se posent alors : comment le goût, ce phénomène réputé totalement subjectif, peut-il nous conduire au jugement et à la révélation du monde commun ? Comment le jugement esthétique permet-il de résister au totalitarisme ? Kant a déjà répondu à la première question : selon lui, le goût est un sens commun ; la condition de l'existence des beaux objets est la «communicabilité » qui procède du goût. Le jugement des spectateurs constitue l'espace public où apparaissent les beaux objets. La réponse à la deuxième question - la capacité de résistance offerte par le jugement esthétique - s'appuie sur l'imagination qui prépare ce jugement réfléchissant. Pour Kant, l'imagination est « la faculté qui rend présent ce qui est absent » (Parménide cité par Arendt 1991) et «transforme un objet en une chose à laquelle je n'ai pas besoin d'être directement confronté, mais que j'ai en un sens intériorisé » (Arendt idem). L'imagination transforme l'objet perçu en objet de représentation auquel je peux réfléchir dans un espace public potentiel ouvert à tous les points de vue. Elle « instaure le recul, le désengagement ou désintéressement requis pour apprécier quelque chose à sa juste valeur » (ibidem). Ainsi, la liberté est liée au pouvoir de l'imagination qui prépare le jugement. On comprend mieux alors l'importance extrême du jugement durant les périodes sombres de l'histoire, lorsqu'il faut s'émanciper des règles admises et des normes légales dont l'application ne permet plus de guider l'action. Dépourvu de cette capacité de jugement, le comptable du camp d'extermination s'habitue à n'être que le trésorier du camp et se prive de toute humanité ; les responsables et les agents de la SNCF font circuler les trains, même chargés de déportés ; les juges appliquent des lois racistes de l'État français... Plus généralement, le jugement instaure cette respiration de l'espace public dont nous parlerons plus loin.

\footnotetext{
${ }^{16}$ Conférences prononcées à la New School for Social Research à New York en automne 1970 (Arendt 1991). Pour l'analyse de ces conférences, nous suivons à la fois le texte d'Arendt et l'excellente interprétation de Myriam Revault d'Allonnes (Arendt idem).

${ }^{17}$ Bien entendu, l'étroite proximité des deux jugements ne tient que parce que la politique est définie par Arendt comme « la mise en commun des paroles et des actes dans un espace d'apparences » (Arendt 1958)
} 
Il reste à préciser deux aspects de cette relecture arendtienne du jugement esthétique kantien. Arendt opère un déplacement important car elle substitue dans sa conception du jugement politique le général à l'universel. Pour Arendt, à la différence de Kant, ce jugement n'est jamais universellement valide. L'universalité est incompatible avec la pluralité qui est la condition fondamentale de possibilité de la politique. Pour Arendt, l'action « correspond à la condition humaine de la pluralité, au fait que ce sont des hommes, et non pas l'homme, qui vivent sur terre et habitent le monde... » (Arendt 1958). C'est pourquoi le jugement politique selon Arendt est général : il autorise la montée en généralité comme désir de politique.

Le deuxième déplacement concerne une proposition de Kant, la primauté des spectateurs sur les acteurs, voir l'exclusion de ces derniers, dans l'établissement d'un espace de jugement. Arendt semble reprendre l'idée, mais pour s'en distancier subtilement. L'accord relatif avec Kant s'explique, comme l'a remarqué Revault d'Allonnes (Arendt 1991), par le refus chez Arendt d'une conséquence possible de l'analogie entre les jugements esthétique et politique : l'esthétisation du politique sous la forme de l'œuvre d'art ${ }^{18}$, elle résiste à cette forme de substitution du faire à l'agir. Cependant, elle prend du recul vis-à-vis de Kant en insistant d'abord sur l'énorme fossé qui sépare acteurs et spectateurs dans la Prusse du XVIIIe siècle finissant (Arendt 1991, $10^{\mathrm{e}}$ conférence). Elle met également l'accent sur ce qui résiste à l'esthétisation du politique : sa conception de l'action comme activité libre qui ne s'épuise jamais dans son produit. Pour Arendt, « la raison d'être de la politique est la liberté et cette liberté est essentiellement expérimentée dans l'action » (Arendt 1961).

Cette lecture de Kant par Arendt permet de considérer le «jugement esthétique » comme un acte contribuant au renouveau politique. Cette action, synonyme de liberté, garantit aussi la contribution de l'imagination à l'espace public. Cet espace public est vif, perpétuellement recréé, aux mains aussi bien des artistes que des spectateurs. Le jugement esthétique fait l'objet d'une négociation qui engage la capacité à monter en généralité à partir d'un point de vue particulier. Ce point de vue s'argumente d'autant mieux que les approches contemporaines de l'art insistent davantage sur le processus de création que la monumentalisation de l'œuvre d'art. Aujourd'hui, l'art tel qu'il s'énonce ressemble fort à la tentative de reformulation de l'espace public par Arendt, comme le montrent les nombreuses expériences «d'art contextuel» liant l'art à l'espace public (Ardenne 2002). En effet, les travaux de nombreux artistes contemporains «incorporent le processus, la durabilité, la pluralité des spectateurs et l'imprédictibilité. En outre, parce qu'ils ne ressemblent pas à ce qui est considéré habituellement comme de l'art, un jugement réfléchissant est requis pour qu'ils retiennent l'attention et pour déterminer s'il s'agit d'art» (Spaid 2003).

\section{Le jugement esthétique offre la possibilité de composer un monde par fragments}

Cependant comment expliquer la montée en généralité d'un processus ? Comment aussi concevoir la place que prennent ces mondes particuliers dans l'instauration d'un monde commun ? Nous discuterons ici brièvement une proposition théorique. Le jugement esthétique propose des fragments ou constitue des propositions fragmentaires qui tendent à participer à l'élaboration d'un espace public, d'un espace discutable, et qui procèdent de logiques non rationnelles. De là, on ne considère plus, sur le plan théorique, l'individu qui fait œuvre, mais le fragment qui met en évidence une certaine conscience du monde : ce qui fait sens,

\footnotetext{
${ }^{18}$ Cette esthétisation du politique - l'État, le parti, le peuple considérés comme œuvre d'art - est qualifiée de national-esthétisme par le philosophe Lacoue-Labarthe 1987 qui y voit une des composantes de l'idéologie nazie.
} 
aujourd'hui. Concrètement, dès lors ce qui fait sens, aujourd'hui, sur le plan politique n'est plus de l'ordre du général et du global, mais du fragmentaire et parcellaire. De l'ouvert et du déchiré. Le fragment est aussi une manière de penser la relation entre le microcosme et le macrocosme. Le fragment vaut pour le système entier ${ }^{19}$ «La totalité fragmentaire est simultanément dans le tout et dans chaque partie. Chaque fragment vaut pour lui-même et pour ce dont il se détache. La totalité, c'est le fragment lui-même dans son individualité achevée. C'est donc la totalité des fragments qui ne compose pas un tout mais qui réplique le tout en chaque fragment. Que la totalité soit présente elle-même en chaque partie, que le tout soit non pas la somme mais la co-présence des parties en tant que co-présence du tout à luimême, c'est ce qui découle de l'individualité du fragment : le tout détaché, c'est l'individu » (Lacoue-Labarthe Nancy 1978). Les fragments installent « la totalité comme pluralité, et son achèvement comme inachèvement de son infinité » (idem).

\section{$\underline{\text { La perspective esthétique revoit le rôle du sujet dans la composition cosmopolitique }}$}

Dans ce sens également, le jugement esthétique donne une place au sujet. Les théoriciens du romantisme allemand (Philippe Lacoue-Labarthe et Jean-Luc Nancy dans L'absolu littéraire, 1978), mais aussi plus tard de nombreux artistes, font de l'artiste un modèle du passage entre l'individu, la fabrication du sujet et le monde. Le monde est analysé comme création, œuvre du sujet. Le monde est une expansion de l'être humain. Il permet d'accroître les possibles en étendant le champ d'action de l'homme. "Comme dans l'idéalisme allemand, la philosophie (le système) du sujet renvoie au système philosophique qui est considéré comme un organisme vivant : le concept est la vie même, la vie est la vie de l'esprit, le système est une totalité organique ». Cependant le romantisme a ses caractères propres : " la vie impliquée ici est la vie belle et l'organisme où elle a lieu et qu'elle anime (mieux vaudrait du reste parler d'organon) est l'œuvre d'art ». Le romantisme suppose donc l'autopoï̀se du sujet et de son monde. Virtuellement donc, tout sujet est créateur même si, pour les romantiques, le passage à la création suppose plus qu'une disposition d'esprit.

\section{Conclusion}

À l'issue de ces premières réflexions, notre démarche se précise. Le poids grandissant de l'esthétique dans l'espace public fournit des pistes pour une démocratisation esthétique des espaces publics. Notons tout d'abord qu'il existe déjà une profonde connivence entre esthétique et politique, ne serait-ce, par exemple, que du côté du droit des paysages (Makowiak 2005). Cependant ce droit qui s'est particulièrement développé en France récemment ne concerne qu'une part limitée de notre problématique, et plus particulièrement la protection de l'environnement. L'intérêt de la confrontation entre esthétique et droit s'est défini comme la possibilité de circonscrire une esthétique devenue objet de droit. Deuxième point donc. Notre propos n'est pas d'ajouter quelque catégorie esthétique aux objets susceptibles d'être défini par le droit. Il n'est pas d'engendrer un mouvement visant à introduire l'esthétique, au sens de considérations sur la beauté, sur la place publique, il est, de manière plus utopique sans doute, de réfléchir aux conditions d'émergence d'une politique (cosmopolitique) faisant intervenir l'esthétique comme la capacité à engendrer de nouvelles figurations du réel, certaines d'entre elles permettant de réconcilier monde naturel et social,

\footnotetext{
${ }^{19}$ Cependant, il faut nuancer ce jugement qui tend à faire du fragment le morceau d'une œuvre ; en effet, le fragment nous renseigne aussi sur la difficulté de dire.
} 
ainsi qu'à comprendre les modes très singuliers qui leur donne droit de cité dans un espace public aujourd'hui essentiellement préoccupé de science et d'expertise. L'une des modalités de formalisation et de saisie d'un concret associée aux vues de l'esprit, la métaphore, constitue une voie puissante et vive invitant à réfléchir à la réception de l'œuvre d'art, mais aussi à la création au sens artistique et banal du terme. Différentes disciplines (de l'herméneutique à la psychologie cognitive en passant par l'informatique) peuvent être intéressées. Pour Arendt (1978) : «Analogies, métaphores et emblèmes sont des fils par lesquels l'esprit retient le monde y compris quand il a perdu tout contact... Ils représentent la garantie de l'unité de l'expérience humaine. En outre, dans un processus de pensée, ils servent de guides pour permettre de trouver notre direction de peur que nous n'errions aveuglément parmi les expériences où nos sens à défaut de connaissance certaine ne peuvent nous guider. Le simple fait que notre cerveau soit capable de trouver de telles analogies, que le monde des apparences nous rappelle des choses qui ne le soient pas, peut être vu comme une sorte de preuve que le l'esprit et corps, la pensée et la sensibilité, l'invisible et le visible, sont faits pour vivre ensemble...» L'esthétisation de l'espace public traduirait ainsi la « recosmisation» du politique, le désir d'un monde commun façonné par l'esthétique et l'habiter. Au cœur de notre conception se trouve la revalorisation de l'expérience mondaine du sujet qui convertit une partie de l'environnement en un monde singulier. Elle permet de réactiver une autre manière, plus impliquée, sensible et imaginative, de connaître l'environnement. C'est autour de la mise en politique de cette part-monde que devrait s'effectuer la reconstruction «bottom-up » des cosmopolitiques. Cependant certaines questions restent en suspens. Comment faire droit aux critiques du jugement esthétique considéré comme trop intellectuel, Selon quels critères peut-on mettre en œuvre le jugement esthétique ? Comment articuler expérience et jugement esthétique ? Comment mieux mettre en relief la question des formes et penser sa prise en compte dans l'espace public ? Elles feront l'objet d'un prochain article sur la politique des formes. Dernier point enfin : esthétique et écologie scientifique sont-elles susceptibles de s'enrichir mutuellement? L'écologie scientifique notamment en ce qui concerne la restauration des écosystèmes peut-elle se nourrir de l'apport formel et sensible des pratiques artistiques ? L'esthétique peut-elle sortir du champ de la représentation pour aller vers celui de l'action ? Tel est, en effet, l'une des pistes que trace l'art contemporain. Succédant au Land Art, l'art écologique prend en considération l'efficience écologique. Les pratiques des éco-artistes permettent un élargissement de la recherche et des actions destinées à transformer une écologie locale par la participation des artistes à un collectif qui comprend des scientifiques et des aménageurs. L'hybridation des pratiques de recherche, la respiration apportée par les artistes, garantissent une créativité plus libre et, plus spécifiquement, une meilleure capacité de combiner l'histoire culturelle et la restauration écologique ${ }^{20}$. Nous venons de commencer une recherche sur ce thème.

20 Cf. la prise en compte du symbolique dans l'écosystème illustrée par le projet «Ulsan Park » de Patricia Johanson présentée dans Spaid 2002.. 


\section{Bibliographie}

Danièle Archibugi, David Held, 1999, Cosmopolitan Democracy. An Agenda for a New World Order, Cambridge UK, Polity Press.

Paul Ardenne, 2002, L'art contextuel. Création artistique en milieu urbain, en situation d'intervention et de participation, Paris, Flammarion.

Hannah Arendt, 1951, The Origins of Totalitarism, New York, Harcourt.

Hannah Arendt, 1961, Between Past and Future, London, Faber \& Faber.

Hannah Arendt, 1958, The Human Condition, Chicago, University of Chicago Press.

Hannah Arendt, 1978, The life of the mind, New York, Harcourt.

Hannah Arendt, 1991 (éd. orig. 1982), Juger. Sur la philosophie politique de Kant, trad. Myriam Revault d'Allonnes, suivi de deux essais interprétatifs par Ronald Beiner et Myriam Revault d'Allonnes, Paris, Seuil, coll. Libre examen.

Henri Atlan, 1979, Entre le cristal et la fumée, Paris, Seuil.

Gaston Bachelard, 1964, The Poetics of Space. Translated by Maria Jolas ( $1^{\mathrm{e}}$ éd. 1957, Paris PUF), New York, Orion Press, 1964, Boston, Beacon Press, 1969.

Yves Barel, 1984, La société du vide, Paris, Seuil.

Ulrich Beck, 2001 (1 $1^{\text {ère }}$ éd. Risikogesellschaft, Suhrkamp Verlag, Frankfurt am Main, 1986), La société du risque. Sur la voie d'une autre modernité, coll. Alto, Aubier.

Vincent Berdoulay, Paolo da Costa Gomes, Jacques Lolive (dir.), (2004), L'espace public à l'épreuve : régressions et renaissances, Bordeaux, Presses de la MSHA.

Vincent Berdoulay, Nicholas Entrikin, 1998, «Lieu et sujet. Perspectives théoriques », L'Espace géographique, 27 (2), p. 75-89.

Augustin Berque, 2000, Ecoumène. Introduction à l'étude des milieux humains, Paris, Belin.

Augustin Berque, «Milieu et identité humaine », à paraître 2004, Annales de Géographie, numéro spécial sur l'identité.

Augustin Berque, 2004, «Ce qui fonde l'éthique environnementale », à paraître dans Diogène Nathalie Blanc, Jacques Lolive, 2004, La part esthétique de la ville, Blanc N., Boullier D., Lolive J., (Dir.) Cosmopolitiques « Aimons la ville » p. 68-74.

Nathalie Blanc, 2003. «La place de l'animal dans les politiques urbaines », Communications 74, Bienfaisante nature, 159-174.

Nathalie Blanc, 2004, De l'écologique en ville, Ethnologie française XXXIV, 4, p. 601-607.

Luc Boltanski, 1990, L'amour et la justice comme compétences, Paris, Métaillié

Luc Boltanski et Laurent Thévenot, 1991, De la justification (Les économies de la grandeur), Paris, Gallimard.

Nicolas Bourriaud, 2001, Esthétique relationnelle, Dijon, Les presses du réel.

Michel Callon, Pierre Lascoumes et Yannick Barthe, 2001, Agir dans un monde incertain : essai sur la démocratie technique, Paris, Seuil.

Barbara Cassin (dir.) 2004, Vocabulaire européen des philosophies. Dictionnaire des intraduisibles, Paris, Éditions du Seuil / Dictionnaires Le Robert.

Lionel Charles, 2001, «Environnement, incertitudes et risque: du pragmatisme aux développements contemporains », Alliage, n48-49.

Eric Dardel, 1990 ( $1^{\mathrm{e}}$ éd. 1952), L’Homme et la Terre. Nature de la réalité géographique, réédition avec commentaires de Pierre Pinchemel et Jean-Marc Besse, Paris, CTHS.

Gilles Deleuze et Félix Guattari, 1980, Mille Plateaux, Paris, Minuit.

Philippe Descola, 2005, Par-delà nature et culture, Paris, Gallimard.

John Dewey, 1980 (1éd. 1934), Art as Experience, New York, Perigee Books. 
Thierry de Duve, 1989, Au nom de l'art. Pour une archéologie de la modernité, Paris, Minuit. Martin Heidegger, 1986 ( $1^{\mathrm{e}}$ éd. 1927), Être et Temps, (traduction française de François Vezin), Paris, Gallimard, coll. nrf.

David Held, 1996, Cosmopolitan Democracy From the Modern State to Cosmopolitan Governance, Stanford University Press.

David Held, 2002, « Globalization and Cosmopolitanism », Logos, Summer 2002, vol 1, 3, p. $1-18$.

Bertrand Heill, 1994, Le sang noir. Chasse et mythe du sauvage en Europe, Flammarion.

Patrick Howard, 2005, « Nurturing Sense of Place Through the Literature of the Bioregion » in Reconstruction, 5.3 (Summer 2005). http://www.reconstruction.ws/053/howard.shtml

Marc Jimenez, 2004, L'esthétique contemporaine, Paris, Klincksieck.

François Jullien, 1996, Traité de l'efficacité, Paris, Grasset.

Emmanuel Kant, 1993, Critique de la faculté de juger ( $1^{\mathrm{e}}$ éd. 1787), Traduction revue et augmentée par Alexis Philonenko, Paris, Vrin.

Emmanuel Kant, 2000, Critique de la faculté de juger (1 ${ }^{\mathrm{e}}$ éd. 1787), Traduction, présentation, bibliographie et chronologie par Alain Renaut, Paris, Garnier-Flammarion.

Emmanuel Kant, 1999, Projet de paix perpétuelle, collection. Traduction (texte allemand en vis-à-vis) de J. Gibelin ( $1^{\mathrm{e}}$ éd. 1795), Paris, Vrin,

Emmanuel Kant, 2002, Idée d'une histoire universelle au point de vue cosmopolitique. $\left(1^{\mathrm{e}}\right.$ éd. 1784), Traduction de Philippe Folliot, édition électronique de la bibliothèque numérique développée en collaboration avec l'Université du Québec à Chicoutimi fondée et dirigée par Jean-Marie Tremblay, bénévole, professeur de sociologie au Cégep de Chicoutimi.

http://www.uqac.uquebec.ca/zone30/Classiques des sciences sociales/classiques/kant emma nuel/idee histoire univ/idee histoire.html

Philippe Lacoue-Labarthe et Jean-Luc Nancy, 1978, L'absolu littéraire. Théorie de la littérature du romantisme allemand, Paris, Seuil, coll. Poétique.

Philippe Lacoue-Labarthe, 1987, La fiction du politique, Paris, Bourgois.

Bruno Latour, 1993, We have never been modern, English translation revised and augmented by Catherine Porter ( $1^{\mathrm{e}}$ éd. 1992, Paris, La Découverte), Harvard University Press, Cambridge, Mass.

Bruno Latour, 1996, Aramis or the Love of Technology, translation by Catherine Porter $\left(1^{\mathrm{e}}\right.$ éd. 1992, Paris, La Découverte), Harvard University Press, Cambridge, Mass.

Bruno Latour, 2004, Politics of Nature - How to Bring the Sciences into Democracy, English translation by Catherine Porter ( $1^{\mathrm{e}}$ éd La Découverte, Paris, 1999), Harvard University Press, Cambridge, Mass.

Emmanuel Levinas, 1982, Ethique et Infini. Dialogues avec Philippe Nemo, Paris, Fayard.

Jacques Lolive, 2004, "Configurations, prises et propositions », Annales de la Recherche Urbaine, $\mathrm{n}^{\circ} 95$.

Jacques Lolive, 2005, « Des forums hybrides à l'esthétisation : controverses et transformations récentes des espaces publics en France », Cahiers de Géographie du Québec numéro spécial Proximité, Conflit, Coopération, (accord de la revue, à paraître décembre 2005).

Jacques Lolive, Olivier Soubeyran (dir), 2006, L'émergence des cosmopolitiques, Paris, La Découverte.

Herbert Reid, Betsy Taylor, «John Dewey's aesthetic ecology of public intelligence and the grounding of civic environmentalism », Ethics and the Environment, 8 (1).

Paul Ricœur, 1975, La métaphore vive, Paris, Le Seuil.

Michel Roux, 2002, Inventer un nouvel art d'habiter. Le ré-enchantement de l'espace, Paris, L'Harmattan. 
David Seamon, 2000, Phenomenology, Place, Environment, and Architecture : A Review of the Litterature in http://www.arch.ksu.edu/seamon/articles/2000 phenomenology review.htm Martin Seel, 1998, «L'esthétique comme partie d'une éthique différentiée. Douze brefs commentaires », Revue canadienne d'esthétique, vol. 2, hiver 98, revue électronique de la Société canadienne d'esthétique http://www.uqtr.ca/AE/vol 2/index.html

Martin Seel, 2004, «La libération de l'esthétique philosophique par Kant», 2004 Bicentenaire de la mort du philosophe Emmanuel Kant, dossier électronique avec éléments bibliographiques, Goethe-Institut Paris http://www.goethe.de/fr/par/kant/frikant.htm

Peter Sloterdijk, 2000, «Finitude et ouverture, vers une éthique de l'espace », in Yves Michaud (dir.), Qu'est-ce que la culture? (Université de tous les savoirs, vol. 6), Paris, Odile Jacob.

Peter Sloterdijk, 2002 (éd originale 1998), Bulles. Sphères I, Paris, Pauvert.

Peter Sloterdijk, 2003, Ni la mort, ni le soleil. Jeu de piste sous forme de dialogues avec Hans Jürgen Heinrichs, Paris, Pauvert.

Olivier Soubeyran, Véronique Barnier, 1985, «Les enjeux du virage aménagiste », Loisir et Société / Society and Leisure, vol. 8, n¹, printemps 1985.

Sue Spaid, 2002, Ecovention, Current Art to Transform Ecologies in http://greenmuseum.org/c/ecovention/intro frame.html

Sue Spaid, 2003, «a political life. Arendtian aesthtics end open systems », Ethics and the Environment, 8 (1).

Isabelle Stengers, 1996-1997, Cosmopolitiques, 7 tomes, Paris, La Découverte / Les Empêcheurs de penser en rond.

Isabelle Stengers, 2002, Penser avec Whitehead. Une libre et sauvage création de concepts, Seuil.

Isabelle Stengers, (à paraître 2006), «La proposition cosmopolitique », in Jacques Lolive et Olivier Soubeyran (dir.), L'émergence des cosmopolitiques, La Découverte

Etienne Tassin, 2003, Un monde commun. Pour une cosmo-politique des conflits, Seuil.

Laurent Thévenot, 1990, «L'action qui convient», dans Patrick Pharo et Louis Quéré (dir.), Les formes de l'action, Raisons pratiques 1, Paris, Éditions de l'École des Hautes Études en Sciences Sociales.

TLFI (Trésor de la Langue Française Informatisé), 2005, in http://atilf.atilf.fr/tlf.htm. Jakob von Uexküll, 1956, Mondes animaux et mondes humains, Paris, Denoël.

Alfred North Whitehead, 1929, Process and Reality, New York: Macmillan. 